\title{
Engineering the Future: A Conceptual Framework for Evolving Engineering Education
}

\author{
Kai Hua Zhuang, Franz Newland \\ Lassonde School of Engineering, York University, Canada \\ kai.zhuang@lassonde.yorku.ca
}

\begin{abstract}
Today, we live in a world of unprecedented challenges, opportunities, and complexities, where advances in technology are transforming the very fabric of our culture, economy, and environment. Engineering education is responsible for preparing students for the unprecedented challenges, opportunities, and complexities of this emerging future. Here, we share our current perspective on the evolution needed in engineering education via a conceptual model we have developed. In particular, we highlight the need for engineers to engage in social agency over technology, which requires them to understand the social context in which they live and work, and explore the kind of ingenuity needed to create a future where technology meets society's need. We use the model to analyze a number of educational initiatives we have developed at the Lassonde School of Engineering.
\end{abstract}

Keywords: Engineering education, Technology \& society, Future

\section{INTRODUCTION}

In the second decade of $21^{\text {st }}$ century, technology is transforming the very fabric of our culture, economy, society, and environment. From Coursera to BitCoin, from artificial organs to artificial intelligences, we as a people are embracing new ways of learning, working, relating, and being. However, despite the undeniable immensity of this transformation, its direction and destination - be it utopic, dystopic, or somewhere in-between - remains unclear. As engineering educators, we have a responsibility to prepare our students for the complexities of this brave new world, so they can contribute positively to our emerging future.

It is our belief that to prepare our students for the future, we must develop an understanding of the role of engineers and also help both engineers and society in general recognize the agency it has over the future that is emerging. We must then evolve our engineering education in alignment with this understanding. Here, we will present a model we have developed based on our current thinking, and a number of educational initiatives we have developed at the Lassonde School of Engineering at York University that are aligned with this perspective. Many of our activities are influenced by our involvement in the Engineering Change Lab - a Canada-wide social lab initiative that is exploring how the unleash the higher potential of the engineering profession in Canada. The model we present is similar to that used in the Change Lab, but we will explore some nuances that have not been considered previously.

\section{ENGINEERS, TECHNOLOGY, AND SOCIETY}

A common perspective is that the engineer, imbued with certain technical expertise and know-how, is responsible for providing technical solutions to societal problems. Implicit in this perspective is the belief that for an engineer to serve his/her function, s/he must consider both technology and society. This was certainly true at the start of the first Industrial revolution, however engineering today is far more heavily skewed towards technology, leading to the often-heard critique that "engineers are great problem solvers that may not be solving the right problems". This may also have led to the growth of nonengineers engaging increasingly in finding technical solutions to problems. Of itself, such a change is not necessarily negative, but it does suggest the need for a redefinition of the role of the engineer and an increased awareness that such a shift is under way. As the educators of future engineers, today's engineering faculty can often be far more focused on delivering technical content, fueled by a belief in the primacy of technical expertise and knowhow, than the social needs for, and implications of, the technical content being taught.

However, it is not enough for engineers to be just technical problem solvers, especially as we continue to transform as a society. Looking back, despite our growing intellectual and technological prowess in recent years, we have failed to address satisfactorily many issues of societal and planetary significance, such as sustainability, poverty, and equity - particularly gender equity. Furthermore, new issues of similar significance have arisen as consequences of technology, such as the aging of population as a result of medical advancements and the loss of jobs due to 
automation. For the next generation of engineers to participate in the co-creation of our future, it is a necessity for them to supplement their technical expertise with broader perspectives, systems capacities, and creative and emotional intelligence. We have illustrated our perspective on the future need of engineers with respect to technology and society in Figure 1A.

\section{ENGINEERS AND INGENUITY}

The word "engineer" shares a linguistic origin with the word "ingenuity", prompting an alternative perspective that the engineer is a person of ingenuity. For us, being ingenious means to act in a way that creates a future different from the status quo (Figure 1C). The action of ingenuity always takes place in the present; a wise action of ingenuity is simultaneously informed by the past and guided by visioning of the future we want to see (Figure 1C).. Our current perspective is that engineering education can all too easily be skewed towards the past, delivering content that is out-of-date, and it is rare to have an engineering curriculum that is connected to the vision of the future, and that develops future engineers with skills not yet seen much in the workforce, but that will be needed in coming years. The engineering profession, while using current technology, may not often inspire us to create a world that is different from the world of today. In this sense, instead of being ingenious, both engineering and engineering education can too often focus on maintaining the status quo. It is our perspective that there is a need for engineers to be more involved in the envisioning of the future we want to see, and be focused on delivering actions of ingenuity in line with this vision. We have illustrated this perspective on engineering and ingenuity in Figure 1B.

\section{MAPPING TODAY'S WORLD}

Combining the previous two perspectives, we have developed a conceptual model for understanding where we are today (Figure 2A) and where we want to be (Figure 2B), both as a profession and as a society. Currently, the engineering profession (including engineering education) may be considered as focusing on technology and the past. Technology research (including those conducted in engineering schools) is focused on technology and the future. Humanities, arts, and spirituality, in our estimation, are often focused on society and the past. This is, in a sense, our illustration of C. P. Snow's "two cultures", although we have added the time axis. Currently, technology and social entrepreneurship serves as the bridge between technology- and society-focused disciplines. It is our perspective that there is more required when visioning the future of our society, shown by the "??" in the upper right quadrant.

\section{ENGINEERING EDUCATION AT LASSONDE}

At the Lassonde School of Engineering, a number of initiatives were developed to introduce our students to broader perspectives, to create more societal relevance, and to nurture their creative, artistic, and emotional capacities. Using the conceptual model presented in the previous section, we have mapped seven of these initiatives (Figure 3 ). We have been involved in the development of initiatives 1-5, and have participated in initiatives 6 and 7. These initiatives are:

1. Passion Project: a course where students pursue self-selected individual or team projects they are passionate out.

2. Renaissance Projects: interdisciplinary team projects organized as one-off projects or as a part of Capstone course.

3. Creativity Workshops: artistic and creative workshops such as painting, pottery, and baking organized as extra-curricular activities.

4. Explorer's Café: open discussion about where we are and where we want to be as a society.

5. Lassonde Futures Lab: a transdisciplinary envisioning of our collective future involving faculty, staff, and students

6. 50/50: an initiative focused on gender and other diversity issues in engineering

7. Bergeron Entreprenuers in Science and Technology: a technology entrepreneurship program with an increased focus on societal relevance

From Figure 3, we can see that our initiatives cover topical areas not addressed by traditional engineering education and technology research, which are concentrated on the technology side of the diagram. A student would receive a much more rounded educational experience by engaging in these initiatives in addition to his/her existing engineering education, therefore by better prepared for the future.

\section{CONCLUSION}

In this paper, we have discussed the need for engineers to engage in social agency over technology, which requires them to understand the social context in which they live and work, and explore the kind of ingenuity needed to create a future where technology meets society's need. We presented our perspectives on the current state and future need of engineering and engineering education using a conceptual model we have developed, and used this model to map seven educational initiatives at Lassonde that addresses the needs we have highlighted. However, we 
recognize that these initiatives are only a starting point to a long and winding journey of transforming engineering education. We hope our conceptual model can be useful for fellow educators in the development, conceptualization, and assessment of their initiatives, and the initiatives we have mapped can be of inspirational value. 
A

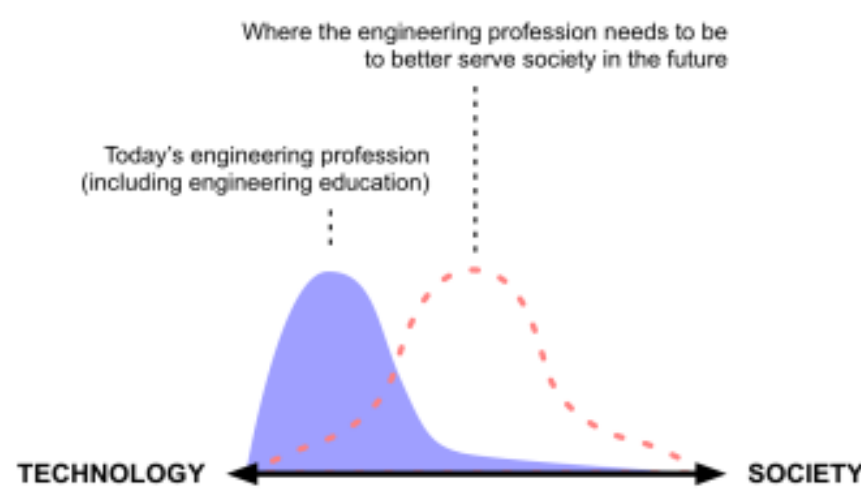

B

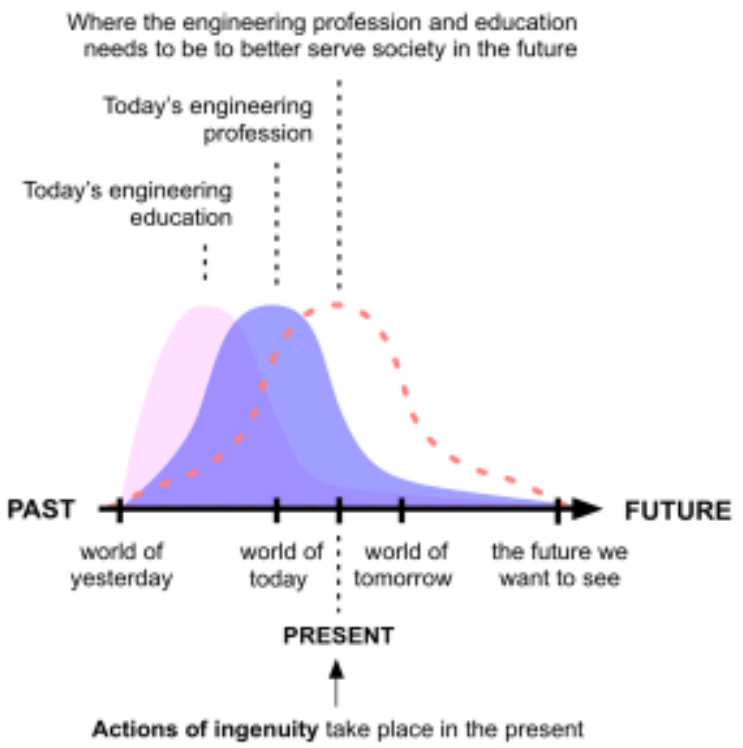

C

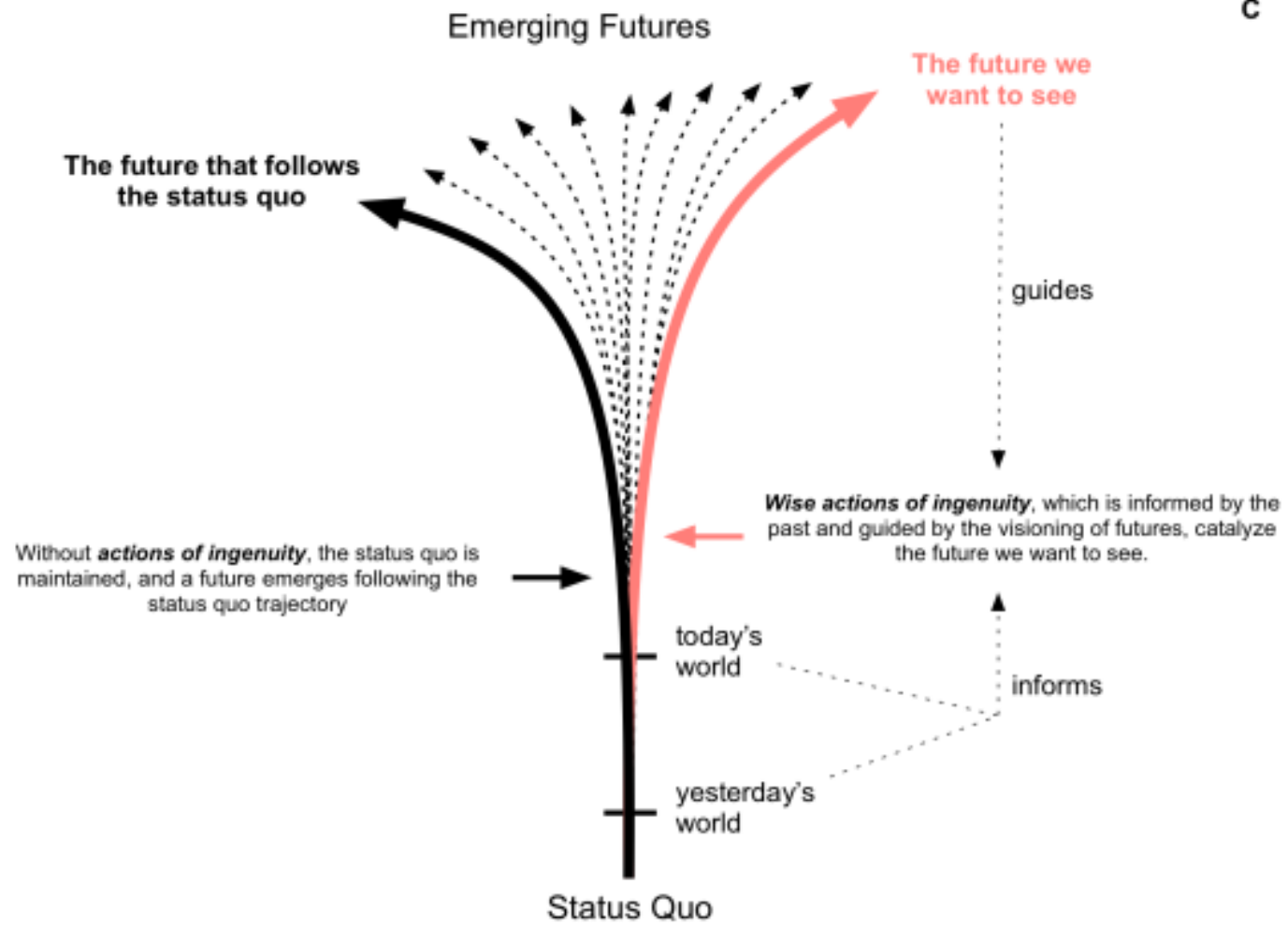

Figure 1. A: The focus of engineering with respect to society and technology. B: The focus of engineering with respect to past, present, and future. C: Ingenuity as actions catalyzing transition from status quo to the future we want to see. 

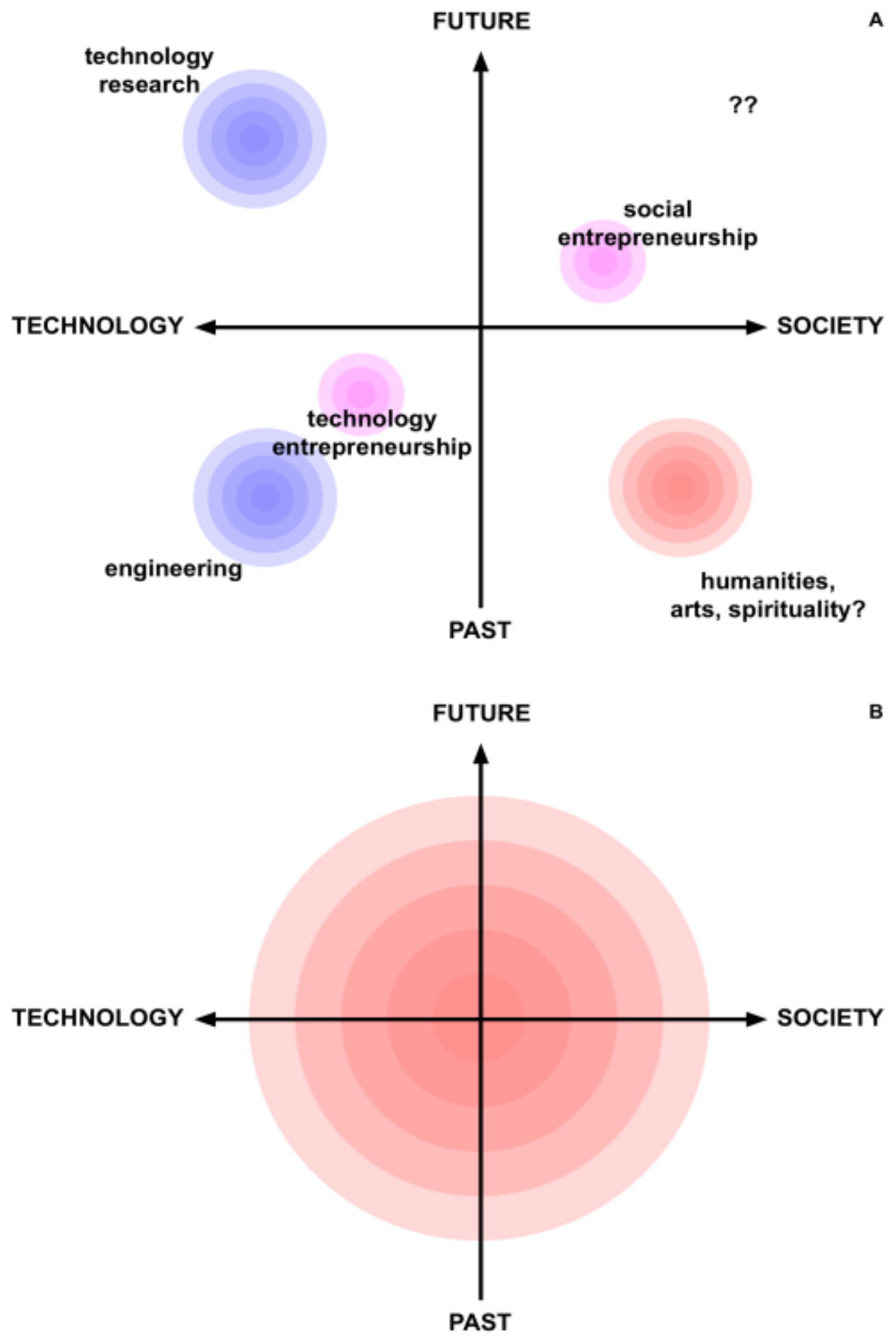

Figure 2. A: Mapping the foci of disciplines in today's world. B: The "ideal spread" of our focus as a profession and as a society. 


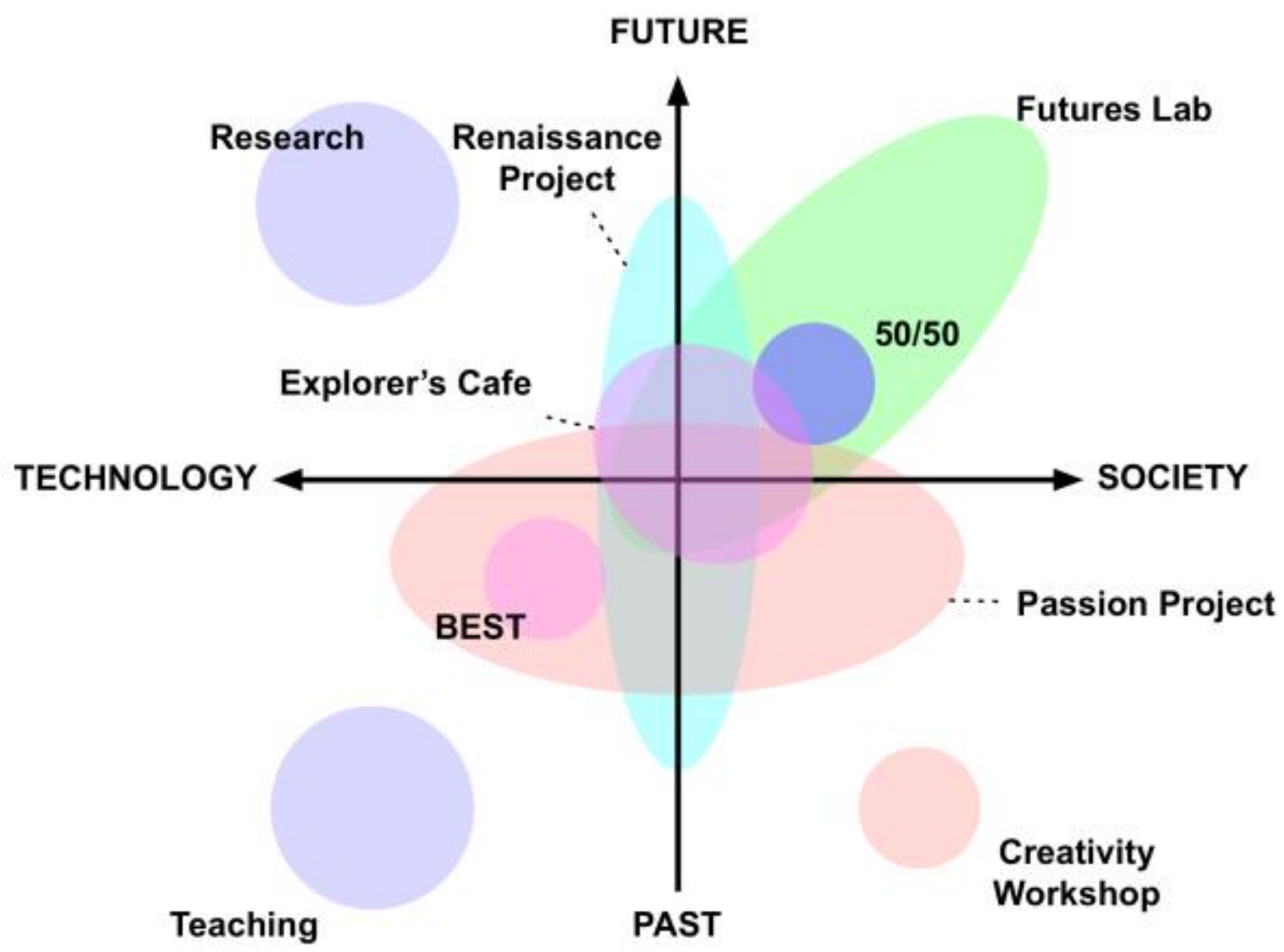

Figure 3. Mapping seven educational initiatives at Lassonde with respect using our conceptual model. 